\title{
SCExAO: A Prototyping Platform for ELTs Extreme-AO
}

\author{
Olivier Guyon ${ }^{\mathrm{a}, \mathrm{b}, \mathrm{c}, \mathrm{d}}$, Julien Lozic ${ }^{\mathrm{c}}$, Nemanja Jovanovic ${ }^{\mathrm{e}}$, and Jared Males ${ }^{\mathrm{b}}$ \\ ${ }^{a}$ Astrobiology Center, National Institutes of Natural Sciences, 2-21-1 Osawa, Mitaka, Tokyo, \\ JAPAN \\ bSteward Observatory, University of Arizona, Tucson, AZ 85721, USA \\ ${ }^{\mathrm{c}}$ National Astronomical Observatory of Japan, Subaru Telescope, National Institutes of \\ Natural Sciences, Hilo, HI 96720, USA \\ ${ }^{\mathrm{d}}$ Breakthrough Initiatives \\ ${ }^{e}$ Caltech Optical Observatories, California Institute of Technology, 1200 E. California \\ Boulevard, MC 11-17, Pasadena, CA 91125, USA
}

\begin{abstract}
Direct imaging and spectroscopy of habitable planets will be possible on ELTs if they can reach $\approx 1 \mathrm{e}-8$ contrast at 10-40 mas separation. This performance level, while conceptually within reach, is several orders of magnitude beyond what current ExAO systems deliver and relies on novel approaches/technologies. On-sky prototyping and validation of these new approaches is essential prior to instrument deployment on ELTs.

The Subaru Coronagraphic Extreme Adaptive Optics (SCExAO) system is a welcoming platform for experimentation and can play a valuable role in this process. New hardware and software approaches can first be validated with the internal source, providing an incremental path to on-sky system-level validation. Thanks to multiple dichroic/filter wheels, light can be directed simultaneously to multiple WFSs and cameras by wavelength or greyscale splitting.

The software environment is highly modular and has been optimized to facilitate deployment of new algorithms. Real-time access to all sensor data and calibrated telemetry is provided. Example code can easily be adapted, removing the burden of coding low-latency hardware communication and calibration. Thanks to accurate and stable system-wide timing, open-loop wavefronts are reconstructed in real-time, allowing users to easily deploy predictive filters and test high speed $(\mathrm{kHz})$ speckle modulation and control schemes. In 2017 , SCExAO's RTC offers 100 Tflops computing power. The software architecture is largely hardware-independent, and open-source development will be shared with other AO systems.
\end{abstract}

Keywords: Adaptive Optics, Coronagraphy, Exoplanets

\section{INTRODUCTION}

Current ground-based adaptive optics ( $\mathrm{AO}$ ) systems can image young massive planets thanks to their strong thermal emission. Current near-IR imaging surveys are most sensitive to planets at large angular separations (typically larger than 0.2 arcsec), where residual starlight is fainter. They are largely blind to sub-Jupiter mass planets that cool too rapidly to remain visible in mature planetary systems.

The upcoming generation of ELTs will allow for a considerably larger sample of giant planets to be imaged, including a large number of Neptune and Jupiter size planets around nearby stars. Their atmospheres will be characterized (spectroscopy) thanks to ELTs' large collecting area and sensitivity.

ELTs will also provide, for the first time, two promising paths to imaging and characterizing habitable planets around nearby stars:

Further author information: (Send correspondence to O.G.)

O.G.: E-mail: oliv.guyon@gmail.com, Telephone: 18182938826 
- Thermal imaging in mid-IR. At $\approx 3-14 \mu m$, temperate habitable planets are sufficiently bright to be imaged against the thermal sky backgound emission. Spectroscopy can reveal multiple molecular species $\left(\mathrm{O}_{3}, \mathrm{NH}_{3}\right.$, $\mathrm{CH}_{4}, \mathrm{CO}, \mathrm{H}_{2} \mathrm{O}, \mathrm{CO}_{2}$ ). Sample size consists of approximately 10 nearby A, F and G type stars, mostly limited by angular resolution.

- Reflected light imaging in visible and near-IR $(\approx 0.5-2.5 \mu \mathrm{m})$. The reflected light contrast for habitable planets orbiting M-type stars, at $\approx 1 \mathrm{e}-8$, is accessible from large ground-based telescopes with extreme adaptive optics. Spectroscopy can reveal $\mathrm{O}_{2}(0.76 \mu \mathrm{m}, 1.27 \mu \mathrm{m}), \mathrm{CH}_{4}, \mathrm{H}_{2} \mathrm{O}, \mathrm{CO}_{2}, \mathrm{CH}_{4}, \mathrm{~N}_{2} \mathrm{O}$. Sample size can reach $\approx 100$ nearby M-type stars, and is strongly dependent on instrument contrast and inner working angle.

In this paper, we focus on the reflected light imaging path.

\section{FUNDAMENTALS OF HIGH CONTRAST IMAGING}

High contrast imaging systems rely on three core functions :

- A coronagraph to provide optical cancellation of starlight diffracted by the telescope's aperture.

- Wavefront control to sense and correct atmospheric turbulence, achieve and maintain high contrast in the focal plane image.

- Post-processing techniques and differential detection techniques to separate starlight from the astrophysical source (planet, disk).

The combination of a coronagraph with wavefront control delivers an image where most starlight has been removed, or at least moved outside the scientific region of interest where planet(s) may be present. The corresponding raw contrast can be augmented by differential detection techniques, which are ultimately limited by photon noise from the residual speckle halo.

In the stellar photon noise limited regime, the signal-to-noise ratio may be expressed as a function of instrument raw contrast $C_{\text {raw }}$, star flux $F_{\text {star }}$, and planet flux $F_{\text {planet }}$ :

$$
S N R=\frac{\alpha F_{\text {planet }}}{\sqrt{C_{\text {raw }} \times F_{\text {star }}}} \times \frac{\sqrt{t} D \sqrt{\beta \pi d \lambda}}{2}
$$

where $\beta$ is the system efficiency $(<1), D$ is the telescope diameter, $d \lambda$ the spectral bandwidth and $t$ the exposure time.

\subsection{SNR coefficient $\alpha$}

The SNR coefficient $\alpha$ in Equ. 1 indicates the fraction of planet light usable for measurement. It is equal to unity for planet detection assuming perfect calibration of residual starlight. In polarimetric differential detection, $\alpha$ is the degree of polarization of planet light.

For spectroscopic characterization, $\alpha$ scales with the integrated equivalent width of the spectral feature to be detected, normalized to the observation spectral bandwidth. If the spectral feature to be measured consists of deep absorption lines covering a small fraction $f$ of the spectral bandwidth, then the signal is the "missing light" in these lines and the noise is the stellar photon noise over the same lines, so $\alpha=\sqrt{f}$. With shallower broader absorption lines, $f<\alpha<\sqrt{f}$; the lower limit corresponds to the extreme case where the spectral feature uniformly attenuates light. 


\subsection{Raw contrast requirement}

As a representative example, we consider a $M 4$ type main sequence star at $5 \mathrm{pc}\left(m_{H}=6.33\right)$ observed in a $20 \%$ wide spectral band around $1.63 \mu \mathrm{m}$ with a $30-\mathrm{m}$ diameter telescope. The intensity contrast for a habitable planet is $F_{\text {planet }} / F_{\text {star }}=3 \mathrm{e}-8$. With a $20 \%$ system efficiency, and a $1 \mathrm{hr}$ exposure time, the SNR is :

$$
S N R_{M 4}=0.0322 \frac{\alpha}{\sqrt{C_{\text {raw }}}}
$$

With $\alpha=1$ (detection), reaching $S N R=10$ within $1 \mathrm{hr}$ requires $C_{\text {raw }}=1.0 \mathrm{e}-5$. For spectroscopy detection of a molecular species, assuming $\alpha=0.1$, and a 10-hr exposure time, the required raw contrast is $C_{\text {raw }}=1.0 \mathrm{e}-6$. The required raw contrast varies with stellar type and distance to the star.

Imaging and spectroscopic characterization of a habitable planets around mid-M-type stars in near-IR with ELTs requires raw contrast around 1e-6. More careful analysis reveals that, for the easiest (closest) targets, a 1e-5 raw contrast may be sufficient, while a 1e-6 raw contrast is required for spectroscopic search of biomarkers around a statistically significant $(>10)$ number of stars.

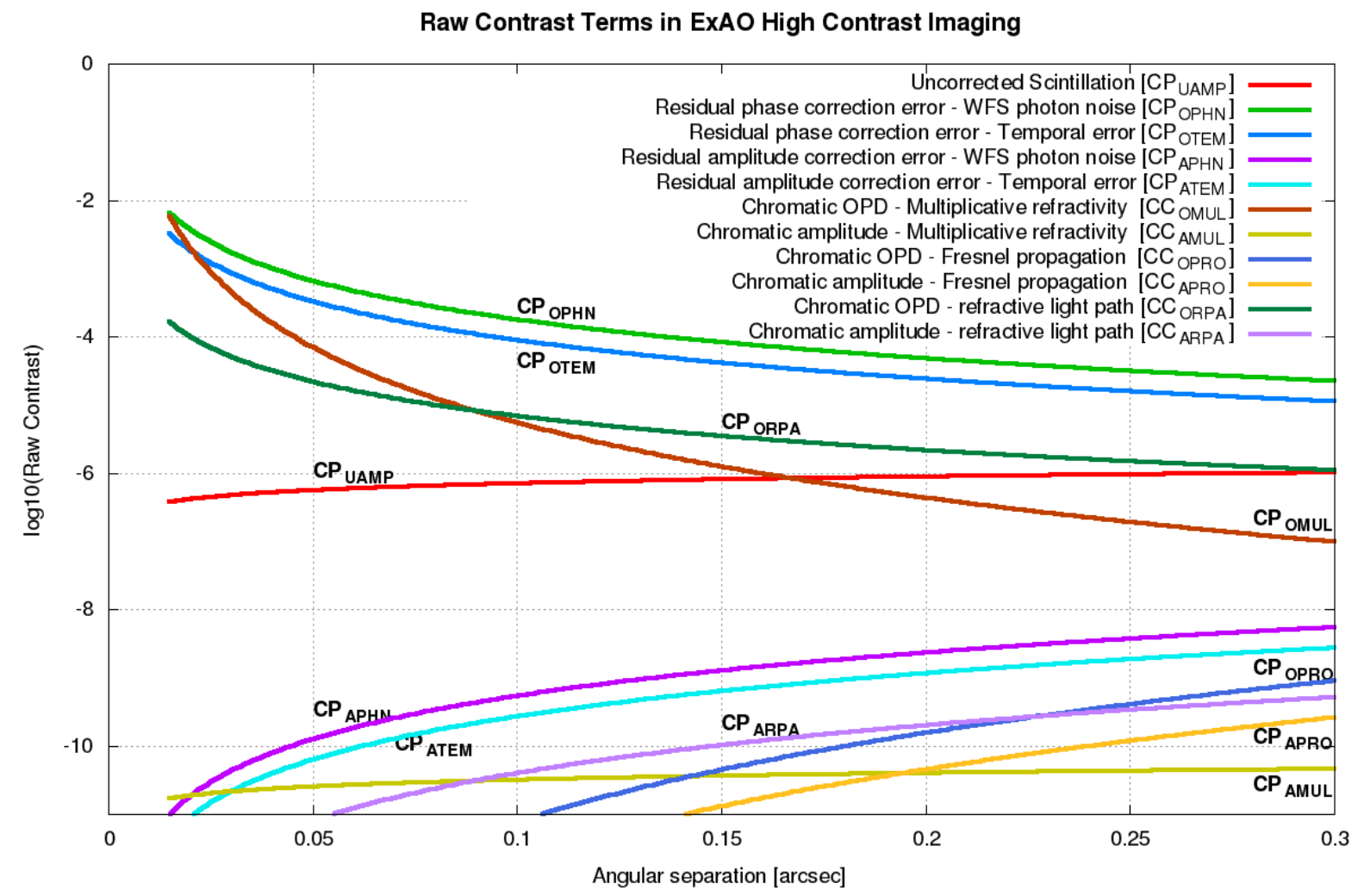

Figure 1. Contribution of main raw contrast terms in an ExAO system. A M4 type main sequence star is observed with a $30 \mathrm{~m}$ diameter telescope. The WFS operates at $0.8 \mu \mathrm{m}\left(m_{I}=8.46\right)$ and high contrast imaging is performed at $1.63 \mu \mathrm{m}$ $\left(m_{H}=6.33\right)$. Atmospheric conditions: wind speed $=10 \mathrm{~m} / \mathrm{s} ; r_{0}=0.15 \mathrm{~m}$ at $\lambda_{0}=0.55 \mu \mathrm{m} ;$ zenith angle $=40$ deg. A Shack-Hartman WFS is assumed.

\section{ACHIEVING DEEP RAW CONTRAST: MAIN CHALLENGES}

The required $\approx 1 \mathrm{e}-6$ raw contrast must be achieved at small angular separation, between 2 and $5 \lambda / D$. This is approximately 3 orders of magnitudes deeper than currently achieved with ExAO systems, and reaching such a contrast level is the most significant challenge to habitable exoplanet imaging and spectroscopy. 
Fig. 1 shows the main raw contrast terms for the M4 star example previously adopted. Five terms dominate, while other terms are not relevant for the $\approx 1 \mathrm{e}-6$ raw contrast goal:

- OPHN: Photon noise in the WFS is the dominant contrast term.

- OTEM: Temporal lag in the AO control loop. An integrator loop is assumed here, with a gain value set to minimize residual wavefront errors in the WFS (sum of OPHN and OTEM terms).

- OMUL: Refractive index chromaticity. Assuming perfect OPD correction in the WFS (at $0.8 \mu \mathrm{m}$ ), a small residual OPD is left at $1.63 \mu \mathrm{m}$.

- OPRA: Due to atmospheric refraction, the light path from the star to the telescope pupil is chromatic, yielding an altitude-dependent chromatic wavefront shear.

- UAMP: Scintillation term.

With the dominant contrast terms identified, priorities for development of ExAO technologies and approaches can be defined.

\subsection{Improving Wavefront Sensing Efficiency and Speed}

Terms OPHN and OTEM dominate the raw contrast error budget. They are fundamentally linked to the wavefront sensing sensitivity, as the control loop adopts the gain that minimizes their sum. To reduce this contribution, three approaches must be developed:

- High sensitivity diffraction-limited wavefront sensing. Conventional WFSs operate in the seeing limited sensitivity regime, and do not take advantage of AO correction at the sensing wavelength. Sensors operating at the diffraction limit (such as a non-modulated Pyramid WFS) are more efficient. The equivalent flux gain is $\left(D / r_{0}\right)^{2}$ for the lowest spatial frequencies, and the combined contribution of terms OPHN and OTEM would be reduced by $\approx 100$ in within 0.1 arcsecond separation.

- Sensor Fusion. Measurements can be combined between multiple sensors operating over a broader wavelength range for increased sensitivity.

- Predictive Control. In a non-predictive control loop, the loop speed needs to be high to reduce the temporal lag term OTEM, increasing the photon noise term OPHN. With predictive control, a zero-lag loop which adequately averages signal to minimize OPHN can be implemented. Early optimistic simulations assuming perfect hardware calibration and frozen flow turbulence suggest $\approx 100 \mathrm{x}$ gain in contrast from predictive control.?

\subsection{Managing Chromaticity}

Terms OPRA and OMUL are due to turbulence chromaticity. While their combined contribution is significant at $\approx 1 \mathrm{e}-4$ contrast level, the corresponding wavefront terms temporal evolution mimics uncorrected atmospheric turbulence and can therefore be significantly reduced with $\mathrm{a} \approx \mathrm{kHz}$ speed control loop. At 30mas separation, the residual 1e-3 contrast is created by aberrations with a 2.5 cycles-per-aperture (CPA) spatial frequency. Assuming $10 \mathrm{~m} / \mathrm{s}$ wind speed, a control loop with $<6 \mathrm{~ms}$ temporal lag is required to reduce the corresponding contrast term by 1000x.

\subsection{Scintillation}

The scintillation term OAMP is near the required 1e-6 contrast level, and can be addressed by either measuring pupil plane intensity in the primary wavefront sensor, or deploying focal-plane wavefront sensing to measure and control speckles. 


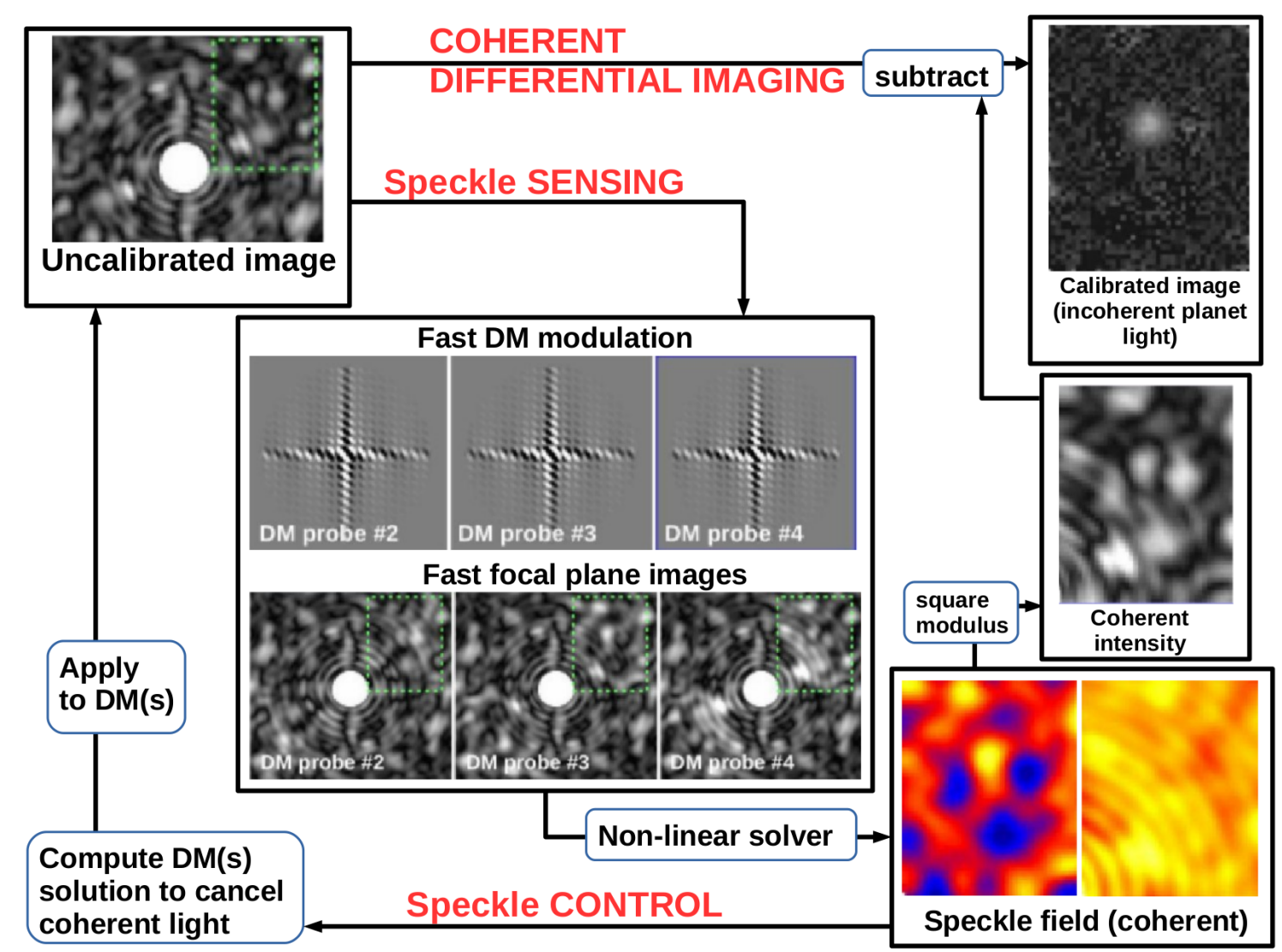

Figure 2. Focal Plane Wavefront Sensing and Control (simulation). The focal plane image (top left) contains residual starlight speckles as well as a planet. The two components cannot be separated in the uncalibrated intensity image alone. The goal of the Speckle Sensing process (center) is to identify coherent starlight in the image by modulating the deformable mirror (DM) and measuring the corresponding images. Several DM probes are applied to the deformable mirror, each diffracting a small fraction of the stellar light. A non-linear solver uses the corresponding images to estimate the coherent speckle filed (bottom right, real and imaginary parts shown). In Coherent Differential Imaging, the reconstructed speckle intensity is subtracted from the uncalibrated image, revealing incoherent light (planet image). In Speckle Control, the reconstructed coherent speckle field is also canceled by DM actuation.

\subsection{Conclusion}

Three promising approaches address the the main limitations to raw contrast:

- Predictive Control can significantly improve AO correction by simultaneoulsy eliminating temporal lag and reducing WFS photon noise. This is particularly valuable for imaging habitable planets around nearby M-type stars, as the stars are not very bright.

- Focal plane wavefront sensing and control largely eliminates non-common path errors, ${ }^{1}$ and addresses chromaticity effects as well as scintillation. As shown in Fig. 2, focal plane wavefront control is also a powerful calibration approach to separate coherent starlight from incoherent planet light.

- Sensor Fusion techniques are required to combine pupil and focal plane measurements across multiple wavelenghts, and can further improve wavefront sensing sensitivity. 


\section{SCEXAO INSTRUMENT OVERVIEW}

\subsection{Hardware configuration}

One key goal for the Subaru Coronagraphic Extreme Adaptive Optics (SCExAO) instrument is to develop and test new approaches in high contrast imaging. Its long term goal is to enable imaging and spectroscopic characterization of habitable planets with ELTs. SCExAO is both a science instrument in operation and a flexible development platform available for the high contrast imaging community.

To support this goal, SCExAO includes a broad range of cameras that can serve as sensors or science instruments, or both. ${ }^{2,3}$ Sensor are listed in Table 1 and shown in the instrument block diagram on Fig. 3.

Table 1. SCExAO imaging sensors.

\begin{tabular}{|l|c|c|l|}
\hline Camera/Module & Wavelength Coverage & Frame Rate & Notes \\
\hline Pyramid WFS & $0.6-0.9 \mu \mathrm{m}$ & $0.5-3.5 \mathrm{kHz}$ & Deep-depletion EMCCD \\
\hline VAMPIRES cameras $(2)$ & $0.6-0.9 \mu \mathrm{m}$ & $\approx 1 \mathrm{kHz}$ & Focal plane EMCCDs \\
\hline FIRST camera & $0.6-0.9 \mu \mathrm{m}$ & $\approx 30 \mathrm{~Hz}$ & spectro-interferometry \\
\hline RHEA IFS & $0.6-0.9 \mu \mathrm{m}, \mathrm{R}=60 \mathrm{k}$ & $<1 \mathrm{~Hz}$ & 3 x3 IFS \\
\hline NIR Viewing Camera & Y-J-H & $\approx 1 \mathrm{kHz}$ & Pupil or focal plane \\
\hline CHARIS IFS & $\mathrm{J}-\mathrm{H}-\mathrm{K}, \mathrm{R}=20-70$ & $\approx 1 \mathrm{~Hz}$ & Main Science Detector \\
\hline MKIDs camera & $\mathrm{J}-\mathrm{H}-\mathrm{K}, \mathrm{R} \approx 10$ & $\approx 5 \mathrm{kHz}$ & Focal plane WFS \\
\hline SAPHIRA imager & Y-J-H-K & $\approx 1-5 \mathrm{kHz}$ & Focal plane HgCaTe APDs \\
\hline Coronagraphic LOWFS & Y-J-H & $\approx 100 \mathrm{~Hz}$ & Coronagraph pointing \\
\hline Photonic Nuller & near-IR & $\approx 100 \mathrm{~Hz}$ & Interferometer \\
\hline Electron Injection camera & Y-J-H & $\approx 1 \mathrm{kHz}$ & Under development \\
\hline Infrared Doppler Sectrograph & $0.97-1.75 \mu \mathrm{m}, \mathrm{R}=70 \mathrm{k}$ & $\approx 1 \mathrm{mn}$ & Currently fed by facility AO \\
\hline
\end{tabular}

The light path includes selectable mirrors and dichroic splitters to direct light to the desired combination of cameras. Nearly all cameras can operate simultaneously, allowing multiple sensors to contribute to wavefront sensing and calibration.

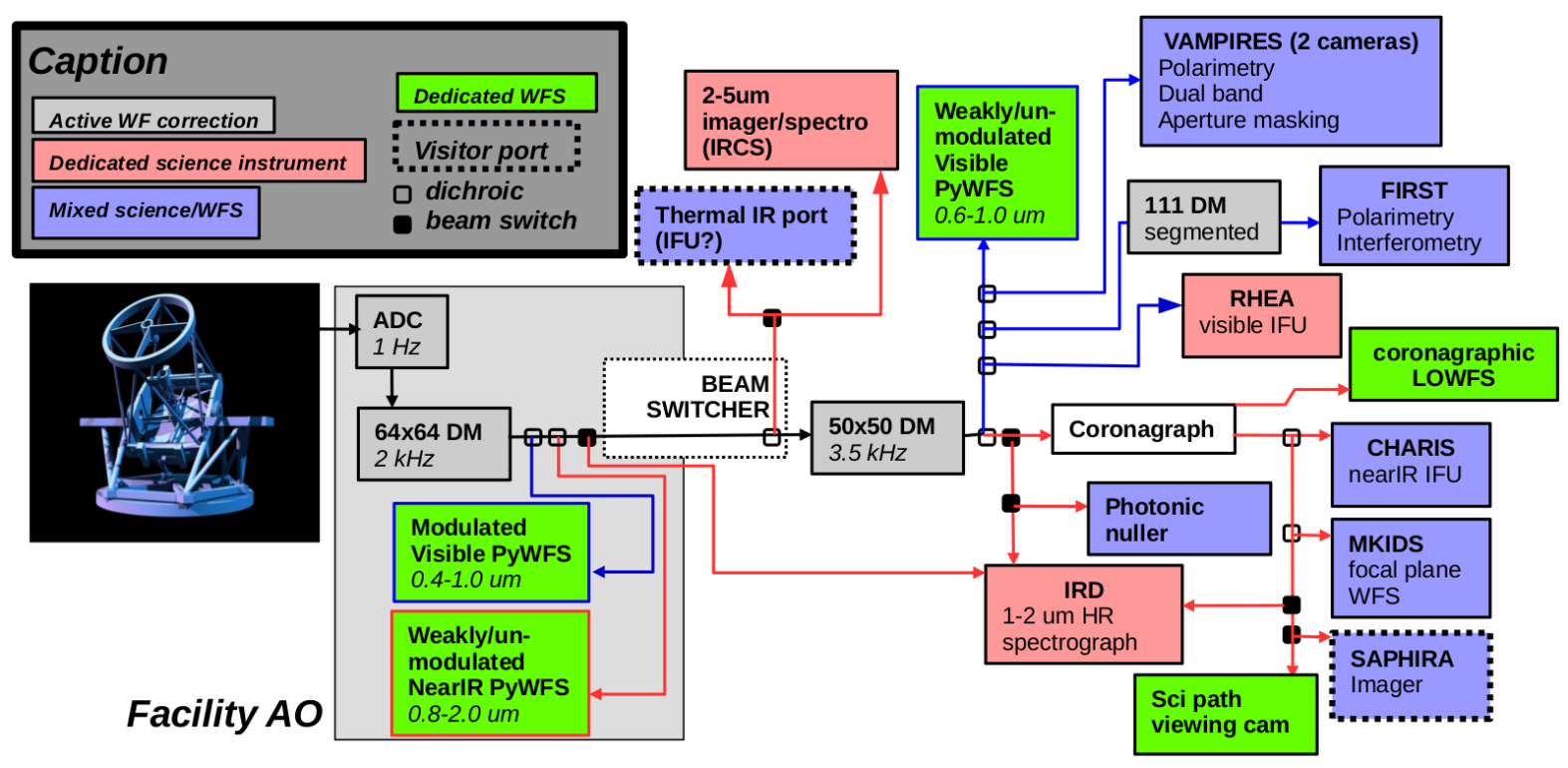

Figure 3. SCExAO Light Path. The facility AO (grey box) is undergoing upgrades - its final configuration is shown here instead of the current system. 


\subsection{Software Architecture}

The SCExAO control software has been developped to support flexible implementation of new wavefront control algorithms. As shown in Fig. 4, all real-time data streams, including raw images, intermediate processing steps and final DM commands, are available to users/developers in real time through a common shared memory-based format. The data format includes multiple semaphores so that each update to a data stream (for example a new image from a wavefront sensor) can trigger an arbitrary number of processes (for example: wavefront reconstruction, data logging to disk). Real-time perfomance and low timing jitter are achieved by system configuration and allocation of time-critical processes to shielded CPU cores. Intensive computations, such as large matrix-vector multiplications, are deployed to one or several of the 10 GPGPUs available, each offering 10 TFLOPS of computing bandwidth.

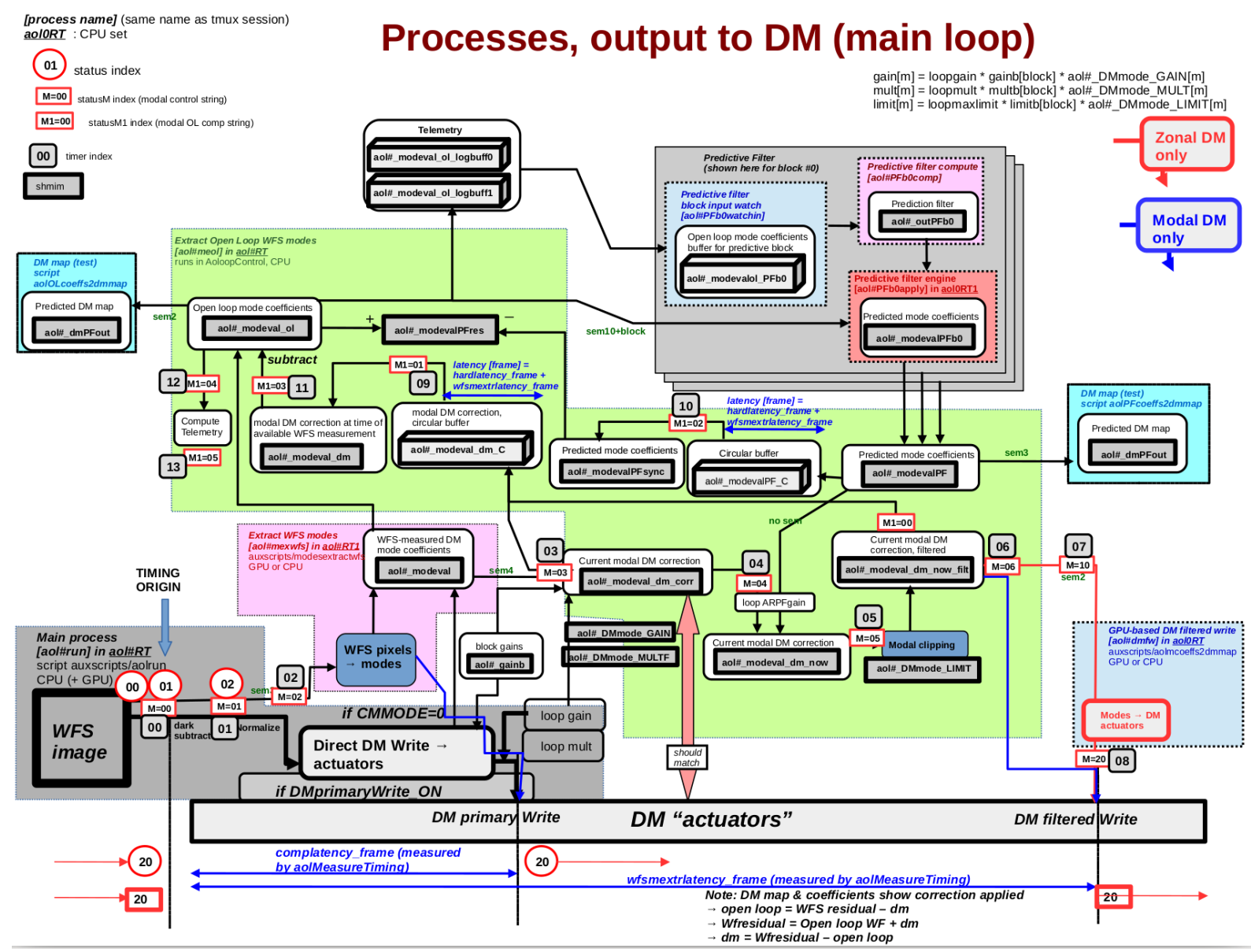

Figure 4. AO control memory block diagram. Each box indicates a data stream, available to users/developers in shared memory. The computing path from WFS image (bottom left) to DM write (bottom right) can be short and simple ("Direct DM Write" path, bottom left), or include modal filtering / open loop reconstruction (light green box) and predictive control (grey box, top right).

The current control software offers high-level functions that are particularly valuable to implement advanced wavefront control algorithms:

- Multiple DM channels provide hooks for users to add DM commands while the wavefront control loop is running. This can be used for diagnostics purposes (adding aberrations, measuring how well they are removed by the control loop), or calibration. 
- Response Matrix (RM) Acquisition in closed loop. The RM can be acquired while the wavefront control loop is running by modulating the DM sufficiently fast.

- Pseudo open-loop real-time wavefront reconstruction. Wavefront measurements and DM control commands are combined with the appropriate time delay to reconstruct the open-loop wavefront entering the system prior to any correction. This feature is used by predictive control algorithms, and is valuable to measure the system performance.

- Links between control loops. Any control loop can, instead of writing directly to a deformable mirror, write to the convergence point of another loop. Control loops can be nested together: for example, speckle modulation uses this feature to send probes to the DM while the primary control loop is running.

- GPU-based predictive control and sensor fusion computing engine. Input signals to the computing engine can be selected to span multiple wavefront sensors, and include recent history for predictive control.

- Telemetry. Any of the real-time telemetry streams can be saved to disk.

\section{CONCLUSIONS}

The upcoming generation of extremely large telescopes (ELTs) will have the sensitivity and angular resolution to image and characterize habitable planets orbiting nearby M-type stars. The measurements will require $\approx 1 \mathrm{e}-5$ raw contrast at a few diffraction widths from the stellar image. While conceptually feasible, this will require new approaches to wavefront sensing and control, including predictive control, sensor fusion and focal plane wavefront sensing/control.

These new approaches are rapidly advancing, and on-sky validation is critical to the success of exoplanet imaging instruments on ELTs. The SCExAO platform provides this capability, and is optimized for flexible implementation of new wavefront control approaches and algorithms. SCExAO's hardware includes multiple sensors and science instruments suitable for development and validation of the key game-changing techniques: sensor fusion, predictive control, and focal plane wavefront control.

The control software has been designed to support research and development in these areas, and will soon be offered to the high contrast imaging community as an open-source resource for extreme-AO development.

\section{ACKNOWLEDGMENTS}

The authors acknowledge support from JSPS Grant-in-Aid for Research \#23340051 and \#26220704.

\section{REFERENCES}

[1] Guyon, O. and Males, J., "Adaptive Optics Predictive Control with Empirical Orthogonal Functions (EOFs)," ArXiv e-prints (July 2017).

[2] Martinache, F., Guyon, O., Jovanovic, N., Clergeon, C., Singh, G., Kudo, T., Currie, T., Thalmann, C., McElwain, M., and Tamura, M., "On-Sky Speckle Nulling Demonstration at Small Angular Separation with SCExAO," PASP 126, 565 (June 2014).

[3] Jovanovic, N., Martinache, F., Guyon, O., Clergeon, C., Singh, G., Kudo, T., Garrel, V., Newman, K., Doughty, D., Lozi, J., Males, J., Minowa, Y., Hayano, Y., Takato, N., Morino, J., Kuhn, J., Serabyn, E., Norris, B., Tuthill, P., Schworer, G., Stewart, P., Close, L., Huby, E., Perrin, G., Lacour, S., Gauchet, L., Vievard, S., Murakami, N., Oshiyama, F., Baba, N., Matsuo, T., Nishikawa, J., Tamura, M., Lai, O., Marchis, F., Duchene, G., Kotani, T., and Woillez, J., "The Subaru Coronagraphic Extreme Adaptive Optics System: Enabling High-Contrast Imaging on Solar-System Scales," PASP 127, 890 (Sept. 2015).

[4] Groff, T. D., Chilcote, J., Kasdin, N. J., Galvin, M., Loomis, C., Carr, M. A., Brandt, T., Knapp, G., Limbach, M. A., Guyon, O., Jovanovic, N., McElwain, M. W., Takato, N., and Hayashi, M., "Laboratory testing and performance verification of the CHARIS integral field spectrograph," in [Ground-based and Airborne Instrumentation for Astronomy VI], Proceedings of the SPIE 9908, 99080O (Aug. 2016). 